\title{
Quadratically constrained quadratic programs on acyclic graphs with application to power
} flow

\author{
Subhonmesh Bose, Student Member, IEEE, Dennice F. Gayme, Member, IEEE, \\ K. Mani Chandy, Fellow, IEEE, and Steven H. Low, Fellow, IEEE
}

\begin{abstract}
This paper proves that non-convex quadratically constrained quadratic programs can be solved in polynomial time when their underlying graph is acyclic, provided the constraints satisfy a certain technical condition. When this condition is not satisfied, we propose a heuristic to obtain a feasible point. We demonstrate this approach on optimal power flow problems over radial networks.
\end{abstract}

\section{Index Terms}

Optimal power flow, distribution circuits, radial networks, energy storage, SDP relaxation, minimum semidefinite rank.

\section{INTRODUCTION}

A quadratically constrained quadratic program (QCQP) is an optimization problem in which the objective function and the constraints are quadratic. In general, QCQPs are non-convex, and therefore lack computationally efficient solution methods. Many engineering problems including

S. Bose is with the Department of Electrical Engineering; S. H. Low and K. M. Chandy are with the Department of Computing and Mathematical Sciences, all at the California Institute of Technology, Pasadena, CA 91125. D. F. Gayme is with the Department of Mechanical Engineering at the Johns Hopkins University, Baltimore, MD 21218 \{boses, mani, slow\}ecaltech.edu, denniceejhu.edu

This work was supported by NSF through NetSE grant CNS 0911041, Southern California Edison, Cisco, and the Okawa Foundation. 
optimal power flow (OPF) can be represented as QCQPs with complex variables. The contribution of this paper is to expand the class of non-convex QCQPs for which globally optimal solutions can be guaranteed.

There is a large literature on optimal or approximate algorithms for QCQPs. One such method employs a convex semidefinite program that is a rank relaxation of the given QCQP. This is commonly referred to as semidefinite relaxation (SDR). Such semidefinite programs are solvable in polynomial time using interior-point methods [1]-[3]. In some instances, an optimal solution of the original QCQP can be recovered from an optimal solution of its SDR. In other cases, SDR provides a way to approximate the solution of a QCQP. Thus, SDR provides a computationally tractable way to approach QCQPs [4], [5]. For example, SDR has been applied to a variety of engineering problems such as MIMO antenna beam-forming [6]-[9], sensor network localization [10], principal component analysis [11] and stability analysis [12]. SDR has also been extensively used in systems and control theory applications [13], [14]. Several authors have investigated exact relaxations, e.g., [15], [16], while others have applied SDR-based approximation techniques to NP-hard combinatorial problems and non-convex QCQPs, e.g., [17]-[19]. The accuracy of these approximations has also been extensively studied, e.g., [20]-[22].

In this paper, we prove a sufficient condition under which QCQPs with underlying acyclic graph structures admit an efficient polynomial time solution using its SDR. We then apply our result to the optimal power flow (OPF) problem on radial networks. OPF is generally a non-convex, NP-hard problem that seeks to minimize some cost function, such as power loss, generation cost and/or user utilities, subject to engineering constraints. Since the original formulation of Carpentier in 1962 [23], various solution techniques have been used for this problem; we refer the reader to [24]-[28] for some surveys. OPF can be cast as a QCQP. The authors in [29], [30] propose to solve its SDR as an approximation. The authors in [31], instead, propose to solve its (convex) Lagrangian dual and provide a sufficient condition under which an optimal solution of the OPF can be recovered from a dual optimal solution. For IEEE test systems and other randomly generated circuits, these approaches have been shown to solve OPF optimally. Recently, OPF over radial networks has been of considerable interest. A checkable sufficient condition has been proved in [32]-[34] where an optimal solution of OPF can be recovered from an optimal solution of its SDR. This paper extends the previously known class of OPF problems that can be solved efficiently. 
The paper is organized as follows. In Section II], we prove a sufficient condition for a nonconvex QCQP over acyclic graphs to be solvable in polynomial time. In Section III, we apply this result to characterize a class of OPF problems over radial networks that can be solved efficiently. In Section $[\mathrm{IV}$ we describe a heuristic method to obtain feasible solutions for QCQPs that do not meet these conditions and thus their optimum solution cannot be directly recovered by solving its SDR. We further apply this technique for the OPF problem and demonstrate through simulations that we can always find a near-optimal feasible point for OPF. We conclude in Section $\mathrm{V}$.

\section{QCQP'S AND SEMIDEFINITE RELAXATION}

Consider the following QCQP with complex variable $x \in \mathbb{C}^{n}$.

\section{Primal problem $P$ :}

$$
\begin{array}{cl}
\underset{x \in \mathbb{C}^{n}}{\operatorname{minimize}} & x^{\mathcal{H}} C x \\
\text { subject to: } & x^{\mathcal{H}} C_{k} x \leq b_{k}, \quad k \in \mathcal{K} .
\end{array}
$$

where $x^{\mathcal{H}}$ denotes the conjugate transpose of $x, C$ is either an $n \times n$ complex positive definite matrix (denoted as $C \succ 0$ ) or a positive semidefinite matrix (denoted as $C \succeq 0$ ), $\mathcal{K}$ is a finite index set, $C_{k}$ is an $n \times n$ complex Hermitian matrix and $b_{k}$ is a scalar for each $k \in \mathcal{K}$.

If the matrices $C_{k}, k \in \mathcal{K}$ are positive semidefinite, then problem $P$ is a convex program and can be solved in polynomial time [35], [36]. If, however, these matrices are not necessarily positive semidefinite, $P$ is non-convex and NP-hard in general. The main result of this paper is the identification of a class of QCQPs that can be solved in polynomial time even though the matrices $C_{k}, k \in \mathcal{K}$ are not necessarily positive semidefinite. This result is applied in the next section to the optimal power flow problem on radial electric networks.

We begin with some notation. Let $[n]:=\{1,2, \ldots, n\}$ and for any matrix $H$, let $H_{i j}$ represent the element corresponding to the $i^{\text {th }}$ row and the $j^{\text {th }}$ column. Define a function $\mathcal{G}$ from QCQP problems to undirected graphs as follows. For a QCQP problem $P$, the undirected graph $\mathcal{G}(P)$ has vertex set $[n]$ and the edge set defined as follows:

$$
(i, j) \text { is an edge in } \mathcal{G}(P) \Longleftrightarrow(i \neq j) \text { and }\left(C_{i j} \neq 0 \text { or }\left[C_{k}\right]_{i j} \neq 0 \text { for some } k \in \mathcal{K}\right)
$$

Since the matrices $C$ and $C_{k}$ are Hermitian, the edges of the graph are undirected. Also, $\mathcal{G}(P)$ has no self-loops from a vertex to itself. We restrict attention to QCQP problems $P$ for which the graph $\mathcal{G}(P)$ is a tree, i.e., it is connected and acyclic. 
For any vector of real numbers $a$, let $a \gg 0$ denote that all elements of $a$ are strictly positive. For any set of complex numbers $U=\left\{u_{1}, \ldots, u_{r}\right\}$, the relative interior of the convex hull [36] of the set is defined as:

$$
\left\{a_{1} u_{1}+a_{2} u_{2}+\ldots a_{r} u_{r} \in \mathbb{C} \mid a \gg 0 \text { and } \sum_{\ell=1}^{r} a_{\ell}=1\right\} .
$$

We restrict our attention to QCQP problems for which the origin of the complex plane does not belong to the relative interior of the convex hull of the set $\left\{C_{i j},\left[C_{k}\right]_{i j}, k \in \mathcal{K}\right\}$ for any edge $(i, j)$ in $\mathcal{G}(P)$. For any edge $(i, j)$ in $\mathcal{G}(P)$, consider the points $C_{i j}$ and $\left[C_{k}\right]_{i j}, k \in \mathcal{K}$ on the complex plane. The convex hull of these points is either a single point, a line segment, or a convex polytope. The condition states that (a) if the hull is a single point, then that point is not the origin, (b) if the hull is a line segment, then the origin is either an extreme point of the line segment or the origin does not lie on the line segment, and (c) if the hull is a convex polytope then the origin is either outside or on the boundary of this polytope. This is illustrated in Figure 1.

We also limit the discussion to QCQPs for which the set of feasible solutions is bounded and has a strictly feasible point, i.e., there exists $x \in \mathbb{C}^{n}$ such that $x^{\mathcal{H}} C_{k} x<b_{k}$ for all $k \in \mathcal{K}$.

To summarize, consider QCQPs that satisfy the following.

Condition 1: (a) $\mathcal{G}(P)$ is connected and acyclic.

(b) For any edge $(i, j)$ in $\mathcal{G}(P)$, the origin is not in the relative interior of the convex hull of $\left\{C_{i j},\left[C_{k}\right]_{i j}, k \in \mathcal{K}\right\}$.

(c) The set of feasible solutions of $P$ is bounded and has a strictly feasible point.

The main result of the paper is the following theorem and its application.

Theorem 1: All QCQPs $P$ that satisfy condition 1 can be solved in polynomial time.

For a continuous optimization problem, we say it can be solved in polynomial time if given any $\zeta>0$, there is an algorithm that finds a feasible solution to the optimization problem with an objective value within $\zeta$ of the theoretical optimum in polynomial time [5], [35], [36]. For QCQPs $P$ that satisfy condition 1, Theorem 1 says that we can construct such a point in polynomial time.

To solve $P$, we use its convex relaxation that can be solved in polynomial time. The relaxation is said to be exact, if there exists an optimal solution of the relaxation that can be mapped to an optimal solution of $P$. An exact (convex) relaxation by itself does not however guarantee that $P$ 

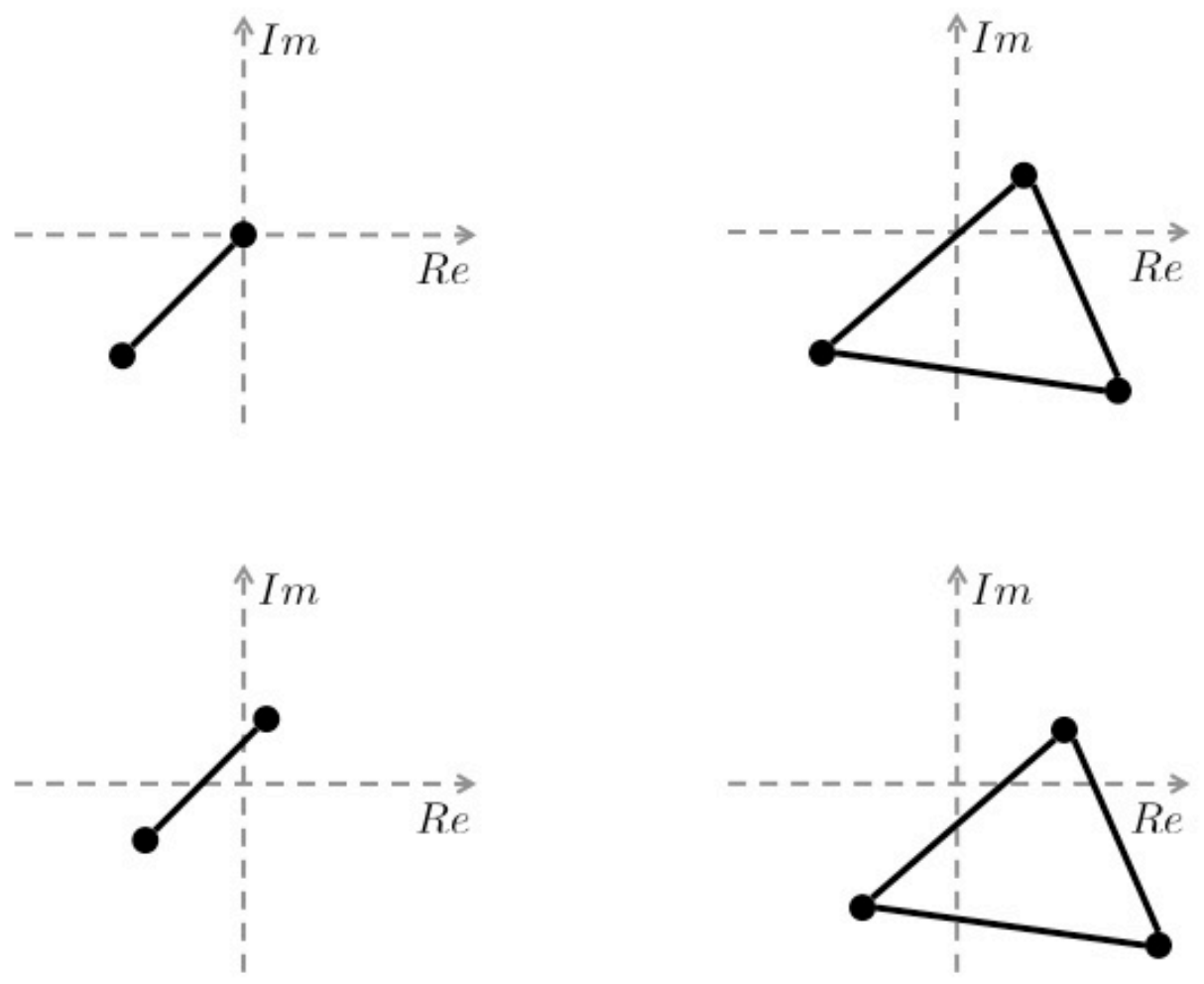

(a) Origin does not belong to the relative interior of the convex hull of these points.
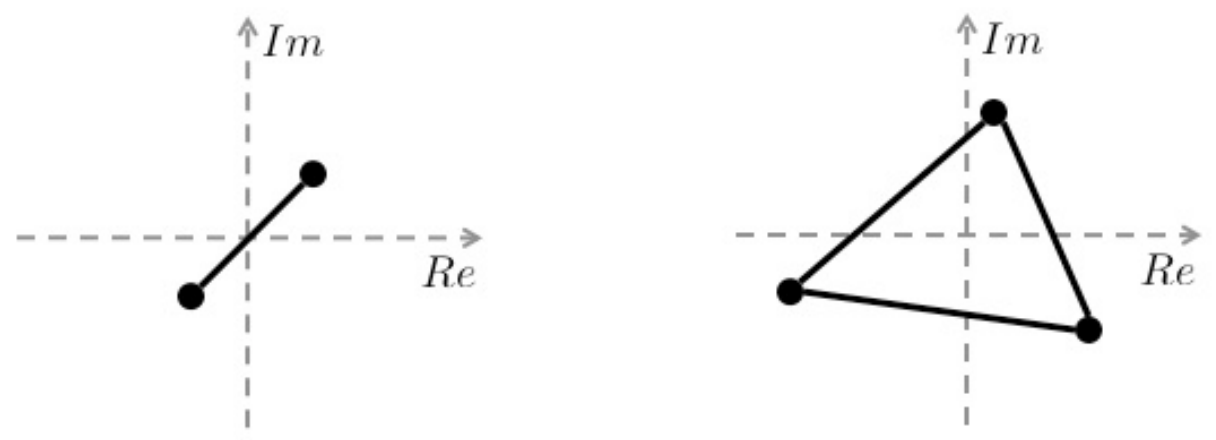

(b) Origin lies in the relative interior of the convex hull of these points.

Fig. 1: Convex hull of $\left\{C_{i j},\left[C_{k}\right]_{i j}, k \in \mathcal{K}\right\}$ in condition 1 b $\mathrm{b}$. 
can be solved in polynomial time. This is the case when the set of optimizers of the relaxation contains solutions that cannot be mapped to a feasible point in $P$ and there may or may not be a polynomial time algorithm to find a correct optimum among that set of optimizers 1 Theorem 1 asserts that when condition 1 holds, not only the convex relaxation of $P$ is exact, but it can also be solved in polynomial time. See Remark 1 at the end of this section for more details.

In the remainder of this section, we prove Theorem 1 for the case where $C$ is positive definite. The proof for the positive semidefinite case is presented in the appendix. Our proof requires the following result on Hermitian matrices, that is presented here and proved in the appendix.

Lemma 2: If $H_{1} \succeq 0$ and $H_{2} \succeq 0$ are two $n \times n$ matrices,

$$
\operatorname{tr}\left(H_{1} H_{2}\right) \geq \rho_{\min }\left[H_{1}\right] \rho_{\max }\left[H_{2}\right] .
$$

where $\rho_{\min }[H]$ and $\rho_{\max }[H]$ respectively denote the minimum and maximum eigenvalues of any Hermitian matrix $H$.

\section{A. Proof of Theorem 1 for $C \succ 0$ :}

For $C \succ 0$, we prove the result more generally by relaxing the condition that the feasible region of $P$ is bounded. Consider the following semidefinite program $R P$ where $W$ is an $n \times n$ complex positive semidefinite matrix.

Relaxed Problem $R P$ :

$$
\begin{array}{cl}
\underset{W \succeq 0}{\operatorname{minimize}} & \operatorname{tr}(C W) \\
\text { subject to: } & \operatorname{tr}\left(C_{k} W\right) \leq b_{k}, \quad k \in \mathcal{K} .
\end{array}
$$

$R P$ is a convex relaxation of $P[5],[36]$. Define $p_{*}$ and $r_{*}$ as the optimum values of the objective functions for problems $P$ and $R P$ respectively.

Lemma 3: $p_{*}, r_{*}$ are finite and $p_{*} \geq r_{*}$. If $W_{*}$ solves $R P$ optimally and rank $W_{*} \leq 1$, then $p_{*}=r_{*}$ and problem $P$ has an exact SDR.

Proof: The objective functions of $P$ and $R P$ are nonnegative and hence $p_{*}$ and $r_{*}$ are finite. Given any feasible solution $x$ of $P, W:=x x^{\mathcal{H}}$ is a feasible solution of $R P$. Hence $R P$

\footnotetext{
${ }^{1}$ If every optimal solution of the relaxation can be mapped to a solution of $P$, then clearly $P$ can be solved in polynomial time. This is sufficient but not a necessary condition for a polynomial time solution.
} 
is feasible and $p_{*} \geq r_{*}$. If rank $W_{*}=0$, then $W_{*}=0$, and an optimal solution to $P$ is $x_{*}=0$, and therefore $r_{*}=p_{*}$. If rank $W_{*}=1$ then $W_{*}$ has a unique decomposition $W_{*}=x_{*} x_{*}^{\mathcal{H}}$, where

$$
r_{*}=\operatorname{tr}\left(C W_{*}\right)=x_{*}^{\mathcal{H}} C x_{*}=p_{*} .
$$

Next, we show that there exists a finite $W_{*}$ that solves $R P$ optimally and has rank $W_{*} \leq 1$.

Let the Lagrange multipliers for the inequalities in (3) be $\lambda_{k} \geq 0$ for each $k \in \mathcal{K}$. Then the Lagrangian dual of $R P$ is

\section{Dual problem $D P$ :}

$$
\begin{array}{ll}
\underset{\lambda \geq 0}{\operatorname{maximize}} & -\sum_{k \in \mathcal{K}} \lambda_{k} b_{k} \\
\text { subject to } & C+\sum_{k \in \mathcal{K}} \lambda_{k} C_{k} \succeq 0 .
\end{array}
$$

For convenience, we introduce the $n \times n$ matrix $A(\lambda)$ defined as:

$$
A(\lambda):=C+\sum_{k \in \mathcal{K}} \lambda_{k} C_{k}
$$

Define a function $\mathcal{F}$ from Hermitian matrices to undirected graphs as follows. For any $n \times n$ Hermitian matrix $H$, the graph $\mathcal{F}(H)$ on the vertex set $[n]$ satisfies

$$
(i, j) \text { is an edge in } \mathcal{F}(H) \Longleftrightarrow i \neq j \text { and } H_{i j} \neq 0 \text {. }
$$

From the definitions of $\mathcal{F}, \mathcal{G}$ and $A(\lambda)$, it follows that for any $\lambda, \mathcal{F}(A(\lambda))$ is a subgraph of $\mathcal{G}(P)$. For some values of $\lambda$ however, edge $(i, j)$ may exist in $\mathcal{G}(P)$ but not in $\mathcal{F}(A(\lambda))$; in this case $\mathcal{F}(A(\lambda))$ is acyclic but may not be connected, and hence it may be a forest of two or more disconnected trees rather than a single connected tree that spans all vertices in the graph. Now, we present a lemma about the connectedness of the graph $\mathcal{F}(A(\lambda))$.

Lemma 4: For all $\lambda \gg 0, \mathcal{F}(A(\lambda))$ is connected.

Proof: Consider any edge $(i, j)$ in $\mathcal{G}(P)$. From condition 1 , the origin is not in the relative interior of the convex hull of $\left\{C_{i j},\left[C_{k}\right]_{i j}, k \in \mathcal{K}\right\}$. Using (4), we have $[A(\lambda)]_{i j} \neq 0$ and hence $(i, j)$ is an edge of $\mathcal{F}(A(\lambda))$. Thus $\mathcal{F}(A(\lambda))$ is identical to $\mathcal{G}(P)$ which is a tree that spans all the vertices of the graph.

Next we characterize the relationship between the optimal points of $R P$ and $D P$. Let $d_{*}$ denote the optimal value of the objective function of $D P$. 
Lemma 5: $r_{*}=d_{*}$ and $R P / D P$ has a finite primal dual optimal point $\left(W_{*}, \lambda_{*}\right)$.

Proof: To prove this, we first show that $D P$ is strictly feasible. At $\lambda=0, \rho_{\min }[A(\lambda)]=$ $\rho_{\min }[C]>0$. For a sufficiently small $\lambda^{\prime} \gg 0, \rho_{\min }\left[A\left(\lambda^{\prime}\right)\right]>0$ and hence $A\left(\lambda^{\prime}\right) \succ 0$. This implies $\lambda^{\prime}$ is a strictly feasible point of $D P$. Also since $P$ is assumed to be strictly feasible, $R P$ is strictly feasible and it has a finite optimum $r_{*}$. The rest follows from Slater's condition [36].

For convenience, define $A_{*}:=A\left(\lambda_{*}\right)$. Now we turn our attention to the graph of $A_{*}$, i.e., $\mathcal{F}\left(A_{*}\right)$ to further analyze the primal dual optimum point $\left(W_{*}, \lambda_{*}\right)$ of $R P / D P$.

Lemma 6: If $\mathcal{F}\left(A_{*}\right)$ is connected then rank $W_{*} \leq 1$.

Proof: We observe that rank $A_{*} \geq n-1$. This follows from a result in the literature [37], [38, Theorem 3.4] and [39. Corollary 3.9] that states that for any $n \times n$ positive semidefinite matrix $H$ where the associated graph $\mathcal{F}(H)$ is a connected acyclic graph (i.e., a tree), rank $H \geq n-1$.

Next we show that rank $W_{*} \leq 1$. The complementary slackness condition for optimality of $\left(W_{*}, \lambda_{*}\right)$ implies

$$
\operatorname{tr}\left(A_{*} W_{*}\right)=0
$$

Let $W_{*}=\sum_{i} \rho_{i} w_{i} w_{i}^{\mathcal{H}}$ be the spectral decomposition of $W_{*}$. Then,

$$
\operatorname{tr}\left(A_{*} W_{*}\right)=\sum_{i} \rho_{i} w_{i}^{\mathcal{H}} A_{*} w_{i}=0
$$

Since $A_{*} \succeq 0$, the eigenvectors $w_{i}$ of $W_{*}$ corresponding to nonzero eigenvalues $\rho_{i}$ are all in the null space of $A_{*}$. The rank of $A_{*}$ is at least $n-1$ and hence its null space has dimension at most 1 , from which it follows that rank $W_{*} \leq 1$.

$\mathcal{F}\left(A_{*}\right)$ can be connected in one of two ways: (a) the origin of the complex plane lies strictly outside the convex hull of the set of points $\left\{C_{i j},\left[C_{k}\right]_{i j} \neq 0, k \in \mathcal{K}\right\}$ for all edges $(i, j)$ in $\mathcal{G}(P)$, or (b) $\lambda_{*} \gg 0$ (from lemma 4). In both cases, lemma 6 guarantees that rank $W_{*} \leq 1$.

If the origin lies on the boundary of the convex hull however, then $\mathcal{F}\left(A_{*}\right)$ may not be connected when $\lambda_{*}$ is not element-wise strictly positive and therefore rank $W_{*} \leq 1$ may not hold. In that case, we cannot obtain an optimum solution of $P$ from the optimum solution of $R P$. We deal with this case where $\mathcal{F}\left(A_{*}\right)$ is disconnected by using a perturbation [40], [41] of $R P / D P$ so that $\mathcal{F}\left(A_{*}\right)$ is connected in the perturbed problem. Then we recover an optimal solution $W_{*}$ for $R P$ such that rank $W_{*} \leq 1$ from the perturbed problem. 
Define the perturbed problems for parameter $\varepsilon>0$ :

Perturbed relaxed problem $R P^{\varepsilon}$ :

$$
\begin{array}{cl}
\underset{W \succeq 0}{\operatorname{minimize}} & \operatorname{tr}(C W)-\varepsilon \sum_{k \in \mathcal{K}}\left[b_{k}-\operatorname{tr}\left(C_{k} W\right)\right] \\
\text { subject to: } & \operatorname{tr}\left(C_{k} W\right) \leq b_{k}, \quad k \in \mathcal{K} .
\end{array}
$$

Perturbed dual problem $D P^{\varepsilon}$ :

$$
\begin{array}{ll}
\underset{\lambda}{\operatorname{maximize}} & -\sum_{k \in \mathcal{K}} \lambda_{k} b_{k} \\
\text { subject to } & A(\lambda) \succeq 0, \lambda_{k} \geq \varepsilon, \quad k \in \mathcal{K} .
\end{array}
$$

For any variable $z$ in the original problem, let $z^{\varepsilon}$ denote the corresponding variable in the perturbed problem with perturbation parameter $\varepsilon$.

Lemma 7: There exists a $\varepsilon_{0}>0$, such that for all $\varepsilon$ in $\left(0, \varepsilon_{0}\right)$ :

1) $r_{*}^{\varepsilon}=d_{*}^{\varepsilon}$ and $R P^{\varepsilon} / D P^{\varepsilon}$ has a finite primal dual optimal point $\left(W_{*}^{\varepsilon}, \lambda_{*}^{\varepsilon}\right)$.

2) $\mathcal{F}\left(A_{*}^{\varepsilon}\right)$ is connected and rank $W_{*}^{\varepsilon} \leq 1$.

Proof: We choose $\varepsilon_{0}$ as follows. For $\varepsilon>0$, observe that

$$
A(\varepsilon \mathbf{1})=C+\varepsilon \sum_{k \in \mathcal{K}} C_{k},
$$

where $\mathbf{1}$ is a vector of all ones of appropriate size. Then $\rho_{\min }[A(\varepsilon \mathbf{1})]$ is a continuous function of $\varepsilon$ that has the value $\rho_{\min }[C]>0$ at $\varepsilon=0$. Choose $\varepsilon_{0}$ sufficiently small such that $\rho_{\min }[A(\varepsilon \mathbf{1})]$ is strictly bounded away from 0 , i.e.,

$$
\min _{\varepsilon \in\left[0, \varepsilon_{0}\right]} \rho_{\min }[A(\varepsilon \mathbf{1})]>0 .
$$

Consider any $\varepsilon$ in $\left(0, \varepsilon_{0}\right)$. The feasible sets of $R P$ and $R P^{\varepsilon}$ are identical. Since $A(\varepsilon \mathbf{1}) \succ 0$ and $W \succeq 0$ for a feasible point $W$ of $R P$ (and $R P^{\varepsilon}$ ),

$$
\operatorname{tr}(C W)-\varepsilon \sum_{k \in \mathcal{K}}\left[b_{k}-\operatorname{tr}\left(C_{k} W\right)\right]=\operatorname{tr}[A(\varepsilon \mathbf{1}) W]-\varepsilon \sum_{k \in \mathcal{K}} b_{k} \geq-\varepsilon_{0}\left|\sum_{k \in \mathcal{K}} b_{k}\right|
$$

and hence $r_{*}^{\varepsilon}$ is finite. $R P^{\varepsilon} / D P^{\varepsilon}$ are strictly feasible and $r_{*}^{\varepsilon}$ is bounded below. The first part of lemma 7 then follows from Slater's condition [36].

To prove the second part of lemma 7, note that $\lambda_{*} \geq \varepsilon \mathbf{1} \gg 0$. Lemmas 4 and 6 applied to $R P^{\varepsilon}$ proves the claim. 
We have shown that for all $\varepsilon$ in a nonempty interval $\left(0, \varepsilon_{0}\right)$, the optimal solution $W_{*}^{\varepsilon}$ of $R P^{\varepsilon}$ has rank at most 1 . Now we analyze the behavior of $W_{*}^{\varepsilon}$ as $\varepsilon$ converges to zero. Let $\left\{\varepsilon_{\ell}\right\}_{\ell=1}^{\infty}$ be a decreasing sequence such that $\varepsilon_{\ell} \rightarrow 0$ as $\ell \rightarrow \infty$. Consider the sequence of matrices $\left\{W_{*}^{\varepsilon_{\ell}}\right\}_{\ell=1}^{\infty}$; every matrix in this sequence has rank at most 1 . In the next lemma we show that this sequence has a convergent subsequence and the limit of this subsequence solves $R P$ optimally.

Lemma 8: $\left\{W_{*}^{\varepsilon}\right\}_{\ell=1}^{\infty}$ has a convergent subsequence. The limit point $\hat{W}$ of this subsequence solves $R P$ optimally and satisfies rank $\hat{W} \leq 1$.

Proof: Consider any $\varepsilon$ in $\left(0, \varepsilon_{0}\right)$. We first show that $W_{*}^{\varepsilon}$ is bounded, independent of $\varepsilon$. For any $W$ in the feasible set of $R P$ (and $R P^{\varepsilon}$ ),

$$
\operatorname{tr}(C W)-\varepsilon \sum_{k \in \mathcal{K}} \underbrace{\left[b_{k}-\operatorname{tr}\left(C_{k} W\right)\right]}_{\geq 0} \leq \operatorname{tr}(C W) .
$$

Minimizing both sides over the feasible set of $R P$ (and $R P^{\varepsilon}$ ), we have

$$
r_{*}^{\varepsilon} \leq r_{*}
$$

that implies

$$
\begin{aligned}
\operatorname{tr}\left[A(\varepsilon \mathbf{1}) W_{*}^{\varepsilon}\right] & =r_{*}^{\varepsilon}+\varepsilon \sum_{k \in \mathcal{K}} b_{k} \\
& \leq r_{*}+\varepsilon_{0}\left|\sum_{k \in \mathcal{K}} b_{k}\right|
\end{aligned}
$$

Also, Lemma 2 implies

$$
\begin{aligned}
\operatorname{tr}\left[A(\varepsilon \mathbf{1}) W_{*}^{\varepsilon}\right] & \geq \rho_{\min }[A(\varepsilon \mathbf{1})] \rho_{\max }\left[W_{*}^{\varepsilon}\right] \\
& \geq \underbrace{\left[\min _{\varepsilon^{\prime} \in\left[0, \varepsilon_{0}\right]} \rho_{\min }\left[A\left(\varepsilon^{\prime} \mathbf{1}\right)\right]\right]}_{>0 \text { by construction. }} \rho_{\max }\left[W_{*}^{\varepsilon}\right] .
\end{aligned}
$$

Combining equations (7) and (8), we obtain a bound for $\rho_{\max }\left[W_{*}^{\varepsilon}\right]$, independent of $\varepsilon$ :

$$
\rho_{\max }\left[W_{*}^{\varepsilon}\right] \leq \frac{r_{*}+\varepsilon_{0}\left|\sum_{k \in \mathcal{K}} b_{k}\right|}{\min _{\varepsilon^{\prime} \in\left[0, \varepsilon_{0}\right]} \rho_{\min }\left[A\left(\varepsilon^{\prime} \mathbf{1}\right)\right]} .
$$

Thus $W_{*}^{\varepsilon}$ is bounded and rank $W_{*}^{\varepsilon} \leq 1$. Since the set of positive semidefinite matrices with rank at most 1 is closed [42], $W_{*}^{\varepsilon}$ lies in a compact set and $\left\{W_{*}^{\varepsilon \ell}\right\}_{\ell=1}^{\infty}$ has a convergent subsequence. Let the limit of this subsequence be $\hat{W}$. Then $\hat{W}$ is feasible for $R P$ and rank $\hat{W} \leq 1$. Next, we prove that $r_{*}=\operatorname{tr}(C \hat{W})$ and hence $\hat{W}$ solves $R P$ optimally. 
From (6),

$$
r_{*}^{\varepsilon}=\operatorname{tr}\left(C W_{*}^{\varepsilon}\right)-\varepsilon \sum_{k \in \mathcal{K}}\left[b_{k}-\operatorname{tr}\left(C_{k} W_{*}^{\varepsilon}\right)\right] \leq r_{*} .
$$

Taking limit over the convergent subsequence of $\left\{W_{*}^{\varepsilon_{\ell}}\right\}_{\ell=1}^{\infty}$, we get $\operatorname{tr}(C \hat{W}) \leq r_{*}$. Also, $r_{*}$ is the optimum value of $R P$ and hence $r_{*} \leq \operatorname{tr}(C \hat{W})$. This completes the proof of lemma 8 .

So far we have shown that $R P$ has a minimizer $\hat{W}$ that satisfies rank $\hat{W} \leq 1$ and $p_{*}=r_{*}$, i.e., SDR of $P$ is exact. But it is, in general, hard to guarantee that solving $R P$ would yield the minimum rank optimizer if the set of optimizers of $R P$ is non-unique. In that case, $R P$ cannot be directly used to solve $P$ in polynomial time. In what follows, we present an algorithm to solve $P$ in polynomial time.

First, solve $R P$ in polynomial time to obtain $r_{*}$. If the associated optimizer $W_{*}$ has rank at most 1 , then construct $x_{*}$ from $W_{*}$ as in lemma 3 . We have then found $x_{*}$ in polynomial time that solves $P$ optimally. If however rank $W_{*}>1$, choose $\varepsilon_{0}$ as given above and solve $R P^{\varepsilon_{0}}$ in polynomial time. For any $\varepsilon$ in $\left(0, \varepsilon_{0}\right)$,

$$
r_{*}^{\varepsilon}=\operatorname{tr}\left(C W_{*}^{\varepsilon}\right)-\varepsilon \sum_{k \in \mathcal{K}}\left[b_{k}-\operatorname{tr}\left(C_{k} W_{*}^{\varepsilon}\right)\right] \leq r_{*} \leq \operatorname{tr}\left(C W_{*}^{\varepsilon}\right),
$$

where the first inequality follows from (6) and the second one follows from the fact that $r_{*}$ is the optimum value of $R P$. Also, comparing the objective function values of $R P^{\varepsilon}$ and $R P^{\varepsilon_{0}}$ at $W_{*}^{\varepsilon}$ and $W_{*}^{\varepsilon_{0}}$ respectively, we have

$$
\sum_{k \in \mathcal{K}}\left[b_{k}-\operatorname{tr}\left(C_{k} W_{*}^{\varepsilon}\right)\right] \leq \sum_{k \in \mathcal{K}}\left[b_{k}-\operatorname{tr}\left(C_{k} W_{*}^{\varepsilon_{0}}\right)\right] .
$$

Combining (10) and (11), we have

$$
\left|r_{*}-\operatorname{tr}\left(C W_{*}^{\varepsilon}\right)\right| \leq \varepsilon \sum_{k \in \mathcal{K}}\left[b_{k}-\operatorname{tr}\left(C_{k} W_{*}^{\varepsilon_{0}}\right)\right] .
$$

Given any $\zeta>0$, choose $\varepsilon$ in $\left(0, \varepsilon_{0}\right)$ such that $\sum_{k \in \mathcal{K}}\left[b_{k}-\operatorname{tr}\left(C_{k} W_{*}^{\varepsilon_{0}}\right)\right] \leq \zeta$. Now solve $R P^{\varepsilon}$ in polynomial time to get $W_{*}^{\varepsilon}$ that satisfies rank $W_{*}^{\varepsilon} \leq 1$ and compute $x_{*}^{\varepsilon}$ from it. Then $x_{*}^{\varepsilon}$ is a feasible point of $P$ and

$$
p_{*} \leq\left(x_{*}^{\varepsilon}\right)^{\mathcal{H}} C\left(x_{*}^{\varepsilon}\right) \leq p_{*}+\zeta .
$$

Also, we have computed $x_{*}^{\varepsilon}$ in polynomial time. This completes the proof of Theorem 1 for the case where $C$ is positive definite. We remark that a perturbation by an arbitrary small $\varepsilon>0$ 
can be represented by treating each perturbed scalar variable as a pair $\left[a, a^{\prime}\right]$ to represent $a+\varepsilon a^{\prime}$ when solving $R P$ using any standard polynomial time algorithm like the interior-point method [1]-[3], where the pairs $\left[a, a^{\prime}\right]$ are ordered lexicographically [43].

The proof extending the theorem to the case where $C$ is positive semidefinite is given in the appendix.

Remark 1: The strict feasibility of $P$ in Condition 1 is required to solve $P$ in polynomial time. If we relax that constraint, it can be shown that there still exists a positive semidefinite matrix $W_{*}$ that solves $R P$ optimally and rank $W_{*} \leq 1$, i.e., $P$ has an exact SDR. There might also be other optimal solutions of $R P$ that do not satisfy the rank condition and hence cannot be mapped to an optimal solution of $P$. Solving for a low-rank optimizer arbitrarily closely in polynomial time is hard to guarantee and is a direction for future work.

\section{Optimal Power Flow: An APPlication}

In this section, we apply the results of Section [I] to the optimal power flow (OPF) problem. We start by summarizing some of the recent results on OPF relaxations in Section III-A. In Section $\amalg I I-B$ we formulate OPF as a QCQP. In Section $\amalg I I-C$ we restrict our attention to OPF over radial networks, which are the networks commonly found in distribution circuits, and use Theorem 1 to characterize a set of conditions under which OPF can be solved efficiently.

\section{A. Prior work}

As previously discussed, OPF can be cast as a QCQP. Various non-linear programming techniques have been applied to the resulting nonconvex problem, e.g., in [44]-[46]. An SDR for OPF has been explored in [29], [30] and their simulations indicate that the SDR provides an exact solution of original OPF for many of the IEEE test systems [47]. The authors in [31], [48] propose to solve the convex Lagrangian dual of OPF and derive a sufficient condition under which an optimal solution of OPF can be recovered from an optimal dual solution. Though SDR recovers a solution to OPF on IEEE test systems, it does not work on all problem instances. Such limitations have been most recently reported in [49], though the nonconvexity of power flow solutions have been studied much earlier, e.g., in [33], [50]-[52].

Recently, a series of work has explored a class of problems where such limitations do not apply due to the network topology. It has been independently reported in [32]-[34] that the SDR 
of OPF is exact for radial networks provided certain conditions on the power flow constraints are satisfied. A different approach to OPF has been explored using the branch flow model, first introduced in [53], [54]. While [55] studies a linear approximation of this model, various relaxations based on second-order cone programming (SOCP) have been proposed in [56]-[59]. Authors in [57]-[59] prove that this relaxation is exact for radial networks when there are no upper bounds on loads, or when there are no upper bounds on voltage magnitudes.

Motivated by the results in [57], a more general branch flow model is introduced in [60] for the power flow analysis and optimization for both radial and meshed networks. The precise relationships between the SOCP relaxations and the SDR for the OPF problem has been recently identified in [61].

\section{B. Problem Formulation}

Consider a power system network with $n$ nodes (buses). The admittance-to-ground at bus $i$ is $y_{i i}$ and the admittance of the line between connected nodes $i$ and $j$ (denoted by $i \sim j$ ) is $y_{i j}=g_{i j}-\mathbf{i} b_{i j}$. We assume both $g_{i j}>0$ and $b_{i j}>0$, i.e., the lines are resistive and inductive. Define the corresponding $n \times n$ admittance matrix $Y$ as

$$
Y_{i j}= \begin{cases}y_{i i}+\sum_{j \sim i} y_{i j}, & \text { if } i=j, \\ -y_{i j}, & \text { if } i \neq j \text { and } i \sim j, \\ 0 & \text { otherwise. }\end{cases}
$$

Remark 2: $Y$ is symmetric but not necessarily Hermitian.

The remaining circuit parameters and their relations are defined as follows.

- $V$ and $I$ are $n$-dimensional complex voltage and current vectors, where $V_{k}, I_{k}$ denote the voltage and the injection current at bus $k \in[n]$ respectively. The voltage magnitude at each bus is bounded as

$$
0<\underline{W}_{k} \leq\left|V_{k}\right|^{2} \leq \bar{W}_{k}, \quad k \in[n] .
$$

- $S=P+\mathbf{i} Q$ is the $n$-dimensional complex power vector, where $P$ and $Q$ respectively denote the real and reactive powers and

$$
S_{k}=P_{k}+\mathbf{i} Q_{k}=V_{k} I_{k}^{\mathcal{H}}, k \in[n] .
$$


- $P_{k}^{D}$ and $Q_{k}^{D}$ are the real and reactive power demands at bus $k \in[n]$, which are assumed to be fixed and given.

- $P_{k}^{G}$ and $Q_{k}^{G}$ are the real and reactive power generation at bus $k$. They are decision variables that satisfy the constraints $\underline{P}_{k}^{G} \leq P_{k}^{G} \leq \bar{P}_{k}^{G}$ and $\underline{Q}_{k}^{G} \leq Q_{k}^{G} \leq \bar{Q}_{k}^{G}$.

Power balance at each bus $k \in[n]$ requires $P_{k}^{G}=P_{k}^{D}+P_{k}$ and $Q_{k}^{G}=Q_{k}^{D}+Q_{k}$, which leads us to define

$$
\begin{aligned}
& \underline{P}_{k}:=\underline{P}_{k}^{G}-P_{k}^{D}, \quad \bar{P}_{k}:=\bar{P}_{k}^{G}-P_{k}^{D} \\
& \underline{Q}_{k}:=\underline{Q}_{k}^{G}-Q_{k}^{D}, \quad \bar{Q}_{k}:=\bar{Q}_{k}^{G}-Q_{k}^{D} .
\end{aligned}
$$

The power injections at each bus $k \in[n]$ are then bounded as

$$
\underline{P}_{k} \leq P_{k} \leq \bar{P}_{k}, \quad \underline{Q}_{k} \leq Q_{k} \leq \bar{Q}_{k} .
$$

The branch power flows and their limits are defined as follows.

- $S_{i j}=P_{i j}+\mathbf{i} Q_{i j}$ is the sending-end complex power flow from node $i$ to node $j$, where $P_{i j}$ and $Q_{i j}$ are the real and reactive power flows respectively. The real power flows are constrained as $\left|P_{i j}\right| \leq \bar{F}_{i j}$ where $\bar{F}_{i j}$ is the line-flow limit between nodes $i$ and $j$ and $\bar{F}_{i j}=\bar{F}_{j i}$.

- $L_{i j}=P_{i j}+P_{j i}$ is the power loss over the line between nodes $i$ and $j$, satisfying $L_{i j} \leq \bar{L}_{i j}$ where $\bar{L}_{i j}$ is the thermal line limit and $\bar{L}_{i j}=\bar{L}_{j i}$. Also, observe that since $L_{i j} \geq 0$, we have $\left|P_{i j}\right| \leq \bar{F}_{i j},\left|P_{j i}\right| \leq \bar{F}_{j i}$ if and only if $P_{i j} \leq \bar{F}_{i j}, P_{j i} \leq \bar{F}_{j i}$.

Let $J_{k}=e_{k} e_{k}^{\mathcal{H}}$ where $e_{k}$ is the $k$-th canonical basis vector in $\mathbb{C}^{n}$. Define $Y_{k}:=e_{k} e_{k}^{\mathcal{H}} Y$. Substituting these expressions into (13) yields

$$
\begin{aligned}
S_{k} & =e_{k}^{\mathcal{H}} V I^{\mathcal{H}} e_{k}=\operatorname{tr}\left(V V^{\mathcal{H}}\left(Y^{\mathcal{H}} e_{k} e_{k}^{\mathcal{H}}\right)\right)=V^{\mathcal{H}} Y_{k}^{\mathcal{H}} V \\
& =(V^{\mathcal{H}} \underbrace{\left(\frac{Y_{k}^{\mathcal{H}}+Y_{k}}{2}\right)}_{=: \Phi_{k}} V)+\mathbf{i}(V^{\mathcal{H}} \underbrace{\left(\frac{Y_{k}^{\mathcal{H}}-Y_{k}}{2 \mathbf{i}}\right)}_{=: \Psi_{k}} V),
\end{aligned}
$$

where $\Phi_{k}$ and $\Psi_{k}$ are Hermitian matrices. Thus, the two quantities $V^{\mathcal{H}} \Phi_{k} V$ and $V^{\mathcal{H}} \Psi_{k} V$ are real numbers; moreover

$$
P_{k}=V^{\mathcal{H}} \Phi_{k} V, \quad Q_{k}=V^{\mathcal{H}} \Psi_{k} V
$$


The real power flow from $i$ to $j$ can be expressed as a quadratic form as follows.

$$
P_{i j}=\operatorname{Re}\left\{V_{i}\left(V_{i}-V_{j}\right)^{\mathcal{H}} y_{i j}^{\mathcal{H}}\right\}=V^{\mathcal{H}} M^{i j} V
$$

where $M^{i j}$ is an $n \times n$ Hermitian matrix. Further details of the OPF problem formulation are provided in the appendix.

The thermal loss of the line connecting buses $i$ and $j$ is

$$
L_{i j}=L_{j i}=P_{i j}+P_{j i}=V^{\mathcal{H}} T^{i j} V
$$

where $T^{i j}=T^{j i}:=M^{i j}+M^{j i} \succeq 0$.

For a Hermitian positive semidefinite $n \times n$ matrix $C$, we have

Optimal power flow problem $O P F$ :

$$
\begin{aligned}
& \underset{V \in \mathbb{C}^{n}}{\operatorname{minimize}} V^{\mathcal{H}} C V \\
& \text { subject to: } \\
& \qquad \underline{P}_{k} \leq V^{\mathcal{H}} \Phi_{k} V \leq \bar{P}_{k}, \quad k \in[n], \\
& \quad \underline{Q}_{k} \leq V^{\mathcal{H}} \Psi_{k} V \leq \bar{Q}_{k}, \quad k \in[n], \\
& \quad \underline{W}_{k} \leq V^{\mathcal{H}} J_{k} V \leq \bar{W}_{k}, \quad k \in[n], \\
& \quad V^{\mathcal{H}} M^{i j} V \leq \bar{F}_{i j}, \quad i \sim j, \\
& V^{\mathcal{H}} T^{i j} V \leq \bar{L}_{i j}, \quad i \sim j,
\end{aligned}
$$

where 17a -117e are respectively constraints on the real and reactive powers, the voltage magnitudes, the line flows and thermal losses. Note that since $T^{i j} \succeq 0$, (16) implies that $P_{i j}+P_{j i} \geq 0$. This means that (17d) holds if and only if $\left|P_{i j}\right| \leq \bar{F}_{i j}$, i.e., (17d) bounds the line flows on both ends.

We do not include line-flow constraints that impose an upper bound on the apparent power $\sqrt{P_{i j}^{2}+Q_{i j}^{2}}$ on each branch $i \sim j$. These constraints are not quadratic in voltages and hence beyond the scope of our model.

Remark 3 (Objective Functions): We consider different optimality criteria by changing $C$ as follows:

- Voltages: $C=\mathcal{I}_{n \times n}$ (identity matrix) where we aim to minimize $\|V\|^{2}=\sum_{k}\left|V_{k}\right|^{2}$. 
- Power loss: $C=\left(Y+Y^{\mathcal{H}}\right) / 2$ where we aim to minimize $\sum_{i} g_{i i}\left|V_{i}\right|^{2}+\sum_{i<j} g_{i j}\left|V_{i}-V_{j}\right|^{2}$.

- Production costs: $C=\sum_{k} c_{k} \Phi_{k}$ where we aim to minimize $\sum_{k} c_{k} P_{k}^{G}, c_{k} \geq 0$.

We assume $C$ is positive semidefinite.

\section{Semidefinite relaxation of OPF over radial networks}

Assume hereafter that $O P F$ is feasible. To conform to the notations of Section [I] replace the constraint in (17a) by the equivalent constraints

$$
\begin{aligned}
V^{\mathcal{H}}\left[\Phi_{k}\right] V & \leq \bar{P}_{k}, \quad k \in[n] \\
V^{\mathcal{H}}\left[-\Phi_{k}\right] V & \leq-\underline{P}_{k}, \quad k \in[n] .
\end{aligned}
$$

Similarly rewrite $(17 \mathrm{~b})$ and $(17 \mathrm{c})$. Then the set of matrices $\left\{C_{k}, k \in \mathcal{K}\right\}$ and the set of scalars $\left\{b_{k}, k \in \mathcal{K}\right\}$ in $O P F$ are defined as

$$
\begin{aligned}
\left\{C_{k}, k \in \mathcal{K}\right\} & :=\left\{\Phi_{k},-\Phi_{k}, \Psi_{k},-\Psi_{k}, J_{k},-J_{k}, k \in[n]\right\} \bigcup\left\{M^{i j}, T^{i j}, i \sim j\right\} \\
\left\{b_{k}, k \in \mathcal{K}\right\} & :=\left\{\bar{P}_{k},-\underline{P}_{k}, \bar{Q}_{k},-\underline{Q}_{k}, \bar{W}_{k},-\underline{W}_{k}, k \in[n]\right\} \bigcup\left\{\bar{F}^{i j}, \bar{L}^{i j}, i \sim j\right\}
\end{aligned}
$$

We limit the discussion to $O P F$ instances where the graph of the power network is a tree $\mathcal{T}$ on $n$ nodes. It can be checked that the graph of the problem $O P F$ satisfies

$$
\mathcal{G}(O P F)=\mathcal{T} \text {. }
$$

Thus condition 11 a holds for $O P F$ over $\mathcal{T}$. In general, condition 11 b does not hold for $O P F$ over $\mathcal{T}$. To illustrate this point, consider an edge $(i, j)$ in $\mathcal{T}$. The admittance of the line joining buses $i$ and $j$ is $g_{i j}-\mathbf{i} b_{i j}$. Then $\left[C_{k}\right]_{i j}, k \in \mathcal{K}$ are given as (details are in the appendix):

(a) $\left[\Phi_{i}\right]_{i j}=-g_{i j} / 2+\mathbf{i} b_{i j} / 2$,

(b) $\left[\Phi_{j}\right]_{i j}=-g_{i j} / 2-\mathbf{i} b_{i j} / 2$,

(c) $\left[\Psi_{i}\right]_{i j}=-b_{i j} / 2-\mathbf{i} g_{i j} / 2$,

(d) $\left[\Psi_{j}\right]_{i j}=-b_{i j} / 2+\mathbf{i} g_{i j} / 2$,

(e) $\left[M^{i j}\right]_{i j}=-g_{i j} / 2+\mathbf{i} b_{i j} / 2$,

(f) $\left[M^{j i}\right]_{i j}=-g_{i j} / 2-\mathbf{i} b_{i j} / 2$,

(g) $\left[T^{i j}\right]_{i j}=\left[T^{j i}\right]_{i j}=-g_{i j}$.

For the objective functions considered, we have (a) Voltages: $C_{i j}=0$, 


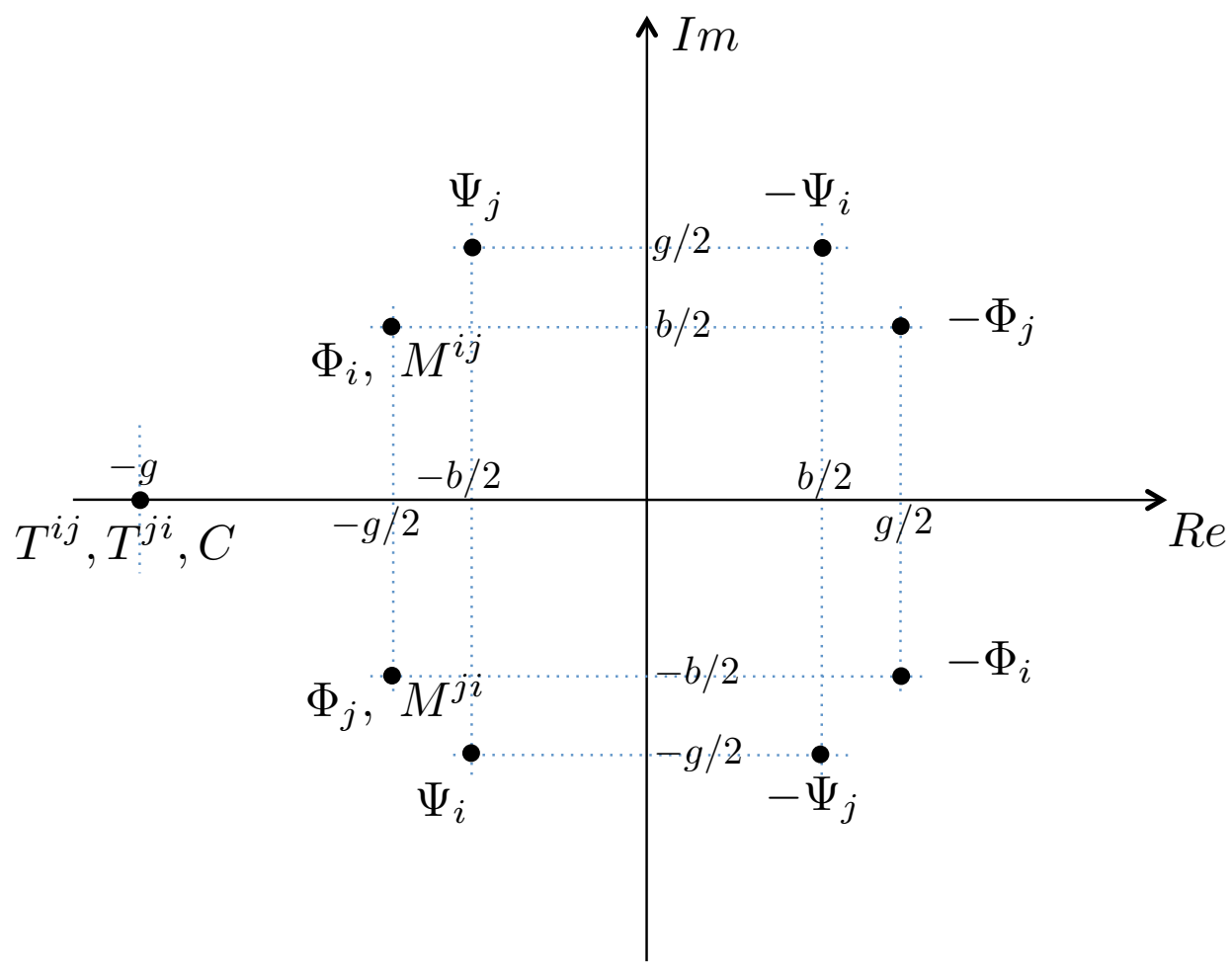

Fig. 2: $C_{i j}$ and non-zero $\left(\left[C_{k}\right]_{i j}, k \in \mathcal{K}\right)$ on the complex plane for $O P F$ for a fixed line $(i, j)$ in tree $\mathcal{T}$.

(b) Power loss: $C_{i j}=-g_{i j}$,

(c) Production costs: $C_{i j}=-g_{i j}\left(c_{i}+c_{j}\right) / 2+\mathbf{i} b_{i j}\left(c_{i}-c_{j}\right) / 2$.

For the purpose of this discussion, consider power-loss minimization as the objective, i.e., $C_{i j}=$ $-g_{i j}$. Also, assume $g_{i j}>b_{i j}>0$. We plot the non-zero $(i, j)$-th entries of the matrices $C_{k}, k \in \mathcal{K}$ and $C$, on the complex plane in Figure 2 and label each point with its corresponding matrix. Clearly if we consider all the points $\left[C_{k}\right]_{i j}, k \in \mathcal{K}$ and $C_{i j}$, the origin of the complex plane lies in the relative interior of the convex hull of these points, i.e., condition 1 b) does not hold.

To apply Theorem 1 to $O P F$, consider the index-set $\tilde{\mathcal{K}} \subseteq \mathcal{K}$ so that condition 1 b holds for $C_{k}, k \in \tilde{\mathcal{K}}$ and $C$. This corresponds to removing certain inequalities in $O P F$, i.e., $b_{k}=+\infty$ for $k \in \mathcal{K} \backslash \tilde{\mathcal{K}}$. For example, removing $-\Phi_{j}$ from the set $\left\{C_{k}, k \in \mathcal{K}\right\}$ corresponds to setting $\underline{P}_{j}=-\infty$.

Condition 1. (c) requires that the feasible set of $O P F$ be bounded. This is always the case 
when $\bar{W}_{k}$ is finite for all buses $k \in[n]$. We discuss the technical condition of strict feasibility in remark 4 ,

Theorem 9: For $\tilde{\mathcal{K}} \subseteq \mathcal{K}$, suppose condition 1 holds for $O P F$ with $C_{k}, k \in \tilde{\mathcal{K}}$ and $C$. Then $O P F$ can be solved in polynomial time.

Remark 4: When $O P F$ is strictly feasible, it can be solved in polynomial time. When strict feasibility does not hold, it follows from remark 1 that $O P F$ still has an exact SDR but we do not guarantee a polynomial time solution.

We explore, through examples, some constraint patterns for $O P F$ over radial networks where a polynomial time solution (for strictly feasible OPF instances) or an exact SDR (for OPFs that may not be strictly feasible) is guaranteed.

Example 1: In Figure 2, consider the $(i, j)$-th elements of the following set of matrices:

$$
\left\{\Phi_{i}, \Phi_{j}, \Psi_{i}, \Psi_{j},-\Psi_{i}, M^{i j}, M^{j i}, T^{i j}=T^{j i}, C\right\} .
$$

The origin of the complex plane lies on the boundary of the convex hull of these points but not in its relative interior. With this set of points, associate a constraint pattern defined as follows. For any point in the diagram that is not a part of this set, the inequality associated with that matrix is removed from $O P F$. For example, the matrices $-\Phi_{j},-\Phi_{i}$ and $-\Psi_{j}$ do not feature on the list of points. Hence,

$$
\underline{P}_{j}=\underline{P}_{i}=\underline{Q}_{j}=-\infty .
$$

This can be generalized to a constraint pattern over $\mathcal{T}$ by removing the lower bounds on real powers at all nodes and the lower bounds on reactive powers at alternate nodes.

Example 2: Suppose $\underline{P}_{k}=\underline{Q}_{k}=-\infty$ for all nodes $k$ in $\mathcal{T}$. This corresponds to considering points only on the left-half plane in Figure 2 for all edges $(i, j)$ in $\mathcal{T}$. Clearly, condition (1) holds in this case. In Figure 2, we assume $g_{i j}>b_{i j}>0$. Regardless of the ordering between $g_{i j}$ and $b_{i j}$ for edges $(i, j)$ in $\mathcal{T}$, the set of points considered in this constraint pattern always lies in the left half of the complex plane.

Removing the lower bounds in real and reactive power can be interpreted as load oversatisfaction, i.e., the real and reactive powers supplied to a node $k$ can be greater than their respective real and reactive power demands $P_{k}^{D}$ and $Q_{k}^{D} . O P F$ on a radial network with load over-satisfaction can be solved efficiently. This result has been reported in [32] $-[34]$. 
Example 3: Consider voltage minimization, i.e., $C=\mathcal{I}_{n \times n}$. In Figure 2, consider the $(i, j)$-th entries of the following set of matrices:

$$
\left\{-\Phi_{i}, \Phi_{j},-\Phi_{j}, \Psi_{i},-\Psi_{j}, C\right\} .
$$

The origin of the complex plane is again on the boundary of the convex hull of these points. The constraint pattern associated with this set of points is

$$
\bar{P}_{i}=\bar{Q}_{j}=\bar{L}_{i j}=\bar{L}_{j i}=\bar{F}_{i j}=\bar{F}_{j i}=+\infty, \quad \text { and } \quad \underline{Q}_{i}=-\infty .
$$

This constraint pattern is consistent with condition 1 b over the edge $(i, j)$ and we can construct a constraint pattern for the OPF problem.

\section{NuMERICAL EXAMPLES}

\section{A. Numerical techniques}

In Section [I] we have identified conditions under which an SDR of a QCQP over an acyclic connected graph can be used to solve the nonconvex problem $P$ efficiently. When these conditions are not satisfied, the QCQP may not be polynomial time computable or have an exact SDR, i.e. $R P$ yields an optimal $W_{*}$ with rank $W_{*}>1$. In that case, $W_{*}$ cannot be mapped to an optimal $x_{*}$ for the problem $P$. In this section we propose a method to construct a feasible solution $\tilde{x}$ for $P$ using such an optimal $W_{*}$ of $R P$. The following relation characterizes the relationship between the optimal solution of $P$ and its value at $\tilde{x}$ :

$$
\text { objective value of } \begin{aligned}
R P \text { at } W_{*} & \leq \text { optimum objective value of } P \\
& \leq \text { objective value of } P \text { at } \tilde{x} .
\end{aligned}
$$

In many practical problems where rank $W_{*}>1$, the principal eigenvalue of $W_{*}$ is orders of magnitude greater than the other eigenvalues. We use the principal eigenvector to search for a "nearby" feasible point of $P$ as follows. Let $w_{*} \in \mathbb{C}^{n}$ be the principal eigenvector of $W_{*}$ and define the starting point $x_{0}$ of the algorithm as

$$
x_{0}:=w_{*} \sqrt{\operatorname{tr}\left(C W_{*}\right)} .
$$

This scaling ensures that the objective value at $x_{0}$ is the optimum objective value of $R P$ at $W_{*}$. If $x_{0}$ satisfies all constraints in $P$, then the algorithm ends with $\tilde{x}=x_{0}$. Otherwise, we construct a sequence of points $\left(x_{1}, x_{2}, \ldots\right)$ where $x_{m+1}$ is constructed from $x_{m}$ as follows. 
1) For $k \in \mathcal{K}$, linearize the function $f_{k}(x)=x^{\mathcal{H}} C_{k} x$ around the point $x_{m}$ and call this function $f_{k}^{(m)}(x)$, i.e.,

$$
f_{k}^{(m)}(x)=x_{m}^{\mathcal{H}} C_{k} x_{m}+2 \operatorname{Re}\left[x_{m}^{\mathcal{H}} C_{k}\left(x-x_{m}\right)\right] .
$$

2) For $k \in \mathcal{K}$, define

$$
s_{k}^{(m)}(x):= \begin{cases}\underline{b}_{k}-f_{k}^{(m)}(x), & \text { if } f_{k}^{(m)}(x) \leq \underline{b}_{k}, \\ 0 & \text { if } \underline{b}_{k} \leq f_{k}^{(m)}(x) \leq \bar{b}_{k}, \\ f_{k}^{(m)}(x)-\bar{b}_{k}, & \text { if } \bar{b}_{k} \leq f_{k}^{(m)}(x) .\end{cases}
$$

We can interpret $s_{k}^{(m)}(x)$ as the amount by which the linearized function $f_{k}^{(m)}$ violates the inequality constraint $\underline{b}_{k} \leq f_{k}^{(m)}(x) \leq \bar{b}_{k}$.

3) Compute $x_{m+1}$ using

$$
\begin{aligned}
x_{m+1}=\underset{x \in \mathbb{C}^{n}}{\arg \min } & \sum_{k \in \mathcal{K}}\left[s_{k}^{(m)}(x)\right]^{2} \\
\text { subject to: } & \left\|x-x_{m}\right\|_{1} \leq \gamma,
\end{aligned}
$$

where $\|.\|_{1}$ denotes the $\ell^{1}$ norm and $\gamma$ is the maximum allowable step-size. This is a parameter for the algorithm and should be chosen such that the linearization $f_{k}^{m}(x)$ is a reasonably good approximation of the quadratic form $f_{k}(x)$ for all $k \in \mathcal{K}$ in the $\ell^{1}$ ball centered around $x_{m}$ with radius $\gamma$.

4) If $x_{m+1}$ satisfies all constraints in $P$, then the algorithm ends with $\tilde{x}=x_{m+1}$.

This heuristic approach either ends at a feasible point $\tilde{x}$ of $P$ or it fails to produce one within a fixed number of iterations. In the next section, we show that this technique performs quite well for numerical $O P F$ examples where the SDR yields an optimal solution $W_{*}$ with rank more than 1 .

\section{B. OPF test examples}

The SDR of $O P F$ and the techniques described in section III are illustrated on a sample distribution circuit from Southern California and randomly generated radial circuits. The semidefinite program is solved in MATLAB using YALMIP [62]. If the solution yielded $W_{*}$ such that rank $W_{*} \leq 1$ then the optimal voltage profile $\left(V_{*}\right)$ to the $O P F$ problem is calculated from $W_{*}=\left(V_{*}\right)\left(V_{*}\right)^{\mathcal{H}}$. If the optimal $W_{*}$ does not satisfy the rank condition, the heuristic approach 
described above is used to find a feasible point of $O P F$. The feasible point obtained may not be optimal for the original problem, so we characterized its sub-optimality by defining the following quantity.

$$
\eta:=\frac{\text { Objective value at heuristically reached feasible point }}{\text { Objective value at optimal point of relaxed problem }}-1 \text {. }
$$

Smaller values of $\eta$ indicate that the feasible point obtained using the algorithm is close to the theoretical optimum of $O P F$.

Throughout this section, let $y=(a, b)$ denote a $y$ drawn from a uniform distribution over the interval $[a, b]$. Using this notation, we describe the test systems used for simulations.

1) SoCal Distribution Circuit: The sample industrial distribution system in Southern California has been previously reported in [57]. It has a peak load of approximately 11.3 MW and installed PV generation capacity of 6.4MW. We modified this circuit by removing the 30MW load at the substation bus (that represented other distribution circuits fed by the same substation) and simulated it with the parameters provided in Table I. To scale the problem correctly, the problem was cast in per unit (p.u.) quantities using base values given in Table 【.

2) Random Test Circuits: These circuits are generated using parameters typical of sparsely loaded rural circuits, as detailed in [63] and employed (with suitable modifications) in [64], [65]. Around $15-60 \%$ of the nodes are assumed to have $2 \mathrm{~kW}$ of PV capacity. The remaining parameters of these systems are described in Table I]

The tests are run with both voltage and power-loss minimization as objective functions. The optimization results are summarized in Table III. For power-loss minimization, we always obtain a rank 1 optimal $W_{*}$.

For voltage minimization, however, we obtain optimal solutions that violate the rank condition. In these cases, the heuristic approach is used to find a feasible point of $O P F$. We construct the solution based on the complex voltage $V_{k}=\left|V_{k}\right| e^{\mathbf{i} \theta_{k}}$ at bus $k \in[n]$. For the heuristic algorithm, define

$$
x:=\left(\left|V_{2}\right|,\left|V_{3}\right|, \ldots,\left|V_{n}\right|, \theta_{2}, \theta_{3}, \ldots, \theta_{n}\right),
$$

and set the parameter $\gamma=+\infty$. In the examples studied, this approach always yields a feasible point within 5 iterations. From Table $\Pi$, the values obtained for $\eta$ indicate that our algorithm 


\begin{tabular}{|c|c|c|}
\hline Test system & SoCal distribution circuit & Random radial networks \\
\hline Number of nodes (n) & 47 & $50-150$ \\
\hline Line impedances $\left(y_{i j}\right)^{-1}$ & [57, Table 1] & $(0.33+\mathbf{i} 0.38) \Omega / \mathrm{km}$, length $=(0.2 \mathrm{~km}, 0.3 \mathrm{~km})$ \\
\hline Voltage limits $\sqrt{\bar{W}_{k}}, \sqrt{\underline{W_{k}}}$ & $1 \pm 0.05$ p.u. at all nodes. & $1 \pm 0.05$ p.u. at all nodes. \\
\hline Real power demand $P_{k}^{D}$ & [57. Table 1] & $(0,4.5 k W)$ \\
\hline Reactive power demand $Q_{k}^{D}$ & Computed with $p . f .=(0.80,0.98)$ lagging & $\left(0.2 P_{k}^{D}, 0.3 P_{k}^{D}\right)$ \\
\hline $\begin{array}{l}\text { Real power gen. limits } \\
\bar{P}_{k}^{G}, \underline{P}_{k}^{G}\end{array}$ & $\begin{array}{l}\text { PV nodes: } \bar{P}_{k}^{G}=(0.2,1.0) \text { times capacity, } \\
\text { Substation node: } \bar{P}_{1}^{G}=10 \mathrm{MW} \\
\text { At all nodes, } \underline{P}_{k}^{G}=0\end{array}$ & $\begin{array}{l}\text { PV nodes: } \bar{P}_{k}^{G}=(0,2 k W) \\
\text { Substation node: } \bar{P}_{1}^{G} \text { scaled with } n \text {. } \\
\text { At all nodes, } \underline{P}_{k}^{G}=0\end{array}$ \\
\hline Reactive power gen. limits & $\bar{Q}_{k}^{G}=0.3 \bar{P}_{k}^{G}, \quad \underline{Q}_{k}^{G}=-0.3 \bar{P}_{k}^{G}$ at all nodes. & $\bar{Q}_{k}^{G}=0.3 \bar{P}_{k}^{G}, \quad \underline{Q}_{k}^{G}=-0.3 \bar{P}_{k}^{G}$ at all nodes. \\
\hline Base quantities & $P_{\text {base }}=1 M W, V_{\text {base }}=12.35 \mathrm{kV}(L-L)$. & $P_{\text {base }}=1 M W, V_{\text {base }}=12.47 \mathrm{kV}(L-L)$. \\
\hline
\end{tabular}

TABLE I: Circuit Parameters for SDR of $O P F$

\begin{tabular}{|l|c|c|c|c|}
\hline Test system & \multicolumn{2}{|c|}{ SoCal distribution circuit } & \multicolumn{2}{c|}{ Random radial networks } \\
\hline Minimize & Power-loss & Voltage & Power-loss & Voltage \\
\hline rank $W_{*}$ & 1 & $\geq 1$ & 1 & $\geq 1$ \\
\hline Mean $\eta$ & N/A & $1.8 \%$ & N/A & $0.5 \%$ \\
\hline Maximum $\eta$ & N/A & $4.1 \%$ & N/A & $1.5 \%$ \\
\hline
\end{tabular}

TABLE II: Summary of simulation results

finds a feasible point of $O P F$ with an objective value close to the theoretical optimum and hence performs well. A general bound on the performance of this heuristic technique, however, remains an open question.

\section{CONCLUSION}

QCQP problems are generally non-convex and NP-hard. This paper proves that a certain class of QCQP problems are solvable in polynomial-time. We have applied this result to the optimal power flow problem and derived a set of conditions under which this nonconvex problem admits an efficient solution. For problems that do not satisfy our sufficient conditions, we provide a heuristic technique to find a feasible solution. Simulations suggest that this method often finds a near-optimal solution for the OPF problem. 


\section{APPENDIX}

\section{A. Proof of lemma 2 .}

The result is restated here for convenience: If $H_{1} \succeq 0$ and $H_{2} \succeq 0$ are two $n \times n$ matrices,

$$
\operatorname{tr}\left(H_{1} H_{2}\right) \geq \rho_{\min }\left[H_{1}\right] \rho_{\max }\left[H_{2}\right] .
$$

Proof: Let the spectral decomposition of $H_{2}$ be

$$
H_{2}=\sum_{i=1}^{n} \rho_{i} u_{i} u_{i}^{\mathcal{H}},
$$

where $\left\|u_{i}\right\|=1$ for all $1 \leq i \leq n$. Then:

$$
\begin{aligned}
\operatorname{tr}\left(H_{1} H_{2}\right) & =\sum_{i=1}^{n} \rho_{i}\left(u_{i}^{\mathcal{H}} H_{1} u_{i}\right) \\
& \geq \sum_{i=1}^{n} \rho_{i} \rho_{\min }\left[H_{1}\right] \\
& \geq \rho_{\min }\left[H_{1}\right] \rho_{\max }\left[H_{2}\right] .
\end{aligned}
$$

B. Proof of Theorem 1 for $C \succeq 0$ :

The sketch of the proof is as follows. Perturb $R P$ so that the matrix in the objective function is positive definite. From our previous analysis this perturbed problem has a finite optimizer that has rank at most 1. Also, this perturbed problem can be solved in polynomial time. Using the solutions from the perturbed problems, we construct an optimum solution of $P$ in polynomial time.

In particular, consider the perturbed problems for $\delta>0$ :

Perturbed primal problem $P^{(\delta)}$ :

$$
\begin{aligned}
\underset{x \in \mathbb{C}^{n}}{\operatorname{minimize}} & x^{\mathcal{H}}(C+\delta \mathcal{I}) x \\
\text { subject to: } & x^{\mathcal{H}} C_{k} x \leq b_{k}, \quad k \in \mathcal{K} .
\end{aligned}
$$

Perturbed relaxed problem $R P^{(\delta)}$ :

$$
\begin{array}{cl}
\underset{W \succeq 0}{\operatorname{minimize}} & \operatorname{tr}[(C+\delta \mathcal{I}) W] \\
\text { subject to: } & \operatorname{tr}\left(C_{k} W\right) \leq b_{k}, \quad k \in \mathcal{K},
\end{array}
$$


where $\mathcal{I}$ is the $n \times n$ identity matrix. For any variable $z$ in $P / R P$, let $z^{(\delta)}$ denote the corresponding variable in $P^{(\delta)} / R P^{(\delta)}$.

The matrix $C+\delta \mathcal{I}$ is positive definite for all $\delta>0$. There exists $W_{*}^{(\delta)} \succeq 0$ that solves $R P^{(\delta)}$ and rank $W_{*}^{(\delta)} \leq 1$. Let the spectral decomposition of $W_{*}^{(\delta)}$ be $W_{*}^{(\delta)}=\left(x_{*}^{(\delta)}\right)\left(x_{*}^{(\delta)}\right)^{\mathcal{H}}$. From Lemma 3, we have

$$
p_{*}^{(\delta)}=\left(x_{*}^{(\delta)}\right)^{\mathcal{H}}(C+\delta \mathcal{I})\left(x_{*}^{(\delta)}\right)=r_{*}^{(\delta)}=\operatorname{tr}\left[(C+\delta \mathcal{I}) W_{*}^{(\delta)}\right] .
$$

The feasible regions of $P$ and $P^{(\delta)}$ are the same and are bounded. Then $x_{*}^{(\delta)}$ lies in a compact space, independent of $\delta$. Taking a sequence $\delta \rightarrow 0$, the corresponding sequence of $x_{*}^{(\delta)}$ has a convergent subsequence. Let the limit point of this subsequence be $\hat{x}$. Then $\hat{x}$ is feasible for $P$. We now show that $\hat{x}$ solves $P$ optimally.

The objective function of $P^{(\delta)}$ increases with $\delta$. Therefore,

$$
p_{*} \leq \hat{x}^{\mathcal{H}} C \hat{x} \leq p_{*}^{(\delta)}=r_{*}^{(\delta)} .
$$

We wish to show that the first inequality is an equality. Suppose on the contrary $p_{*}<\hat{x}^{\mathcal{H}} C \hat{x}$. Let $x_{*}^{\prime}$ be any finite optimizer of $P$. Then,

$$
p_{*}=\left(x_{*}^{\prime}\right)^{\mathcal{H}} C\left(x_{*}^{\prime}\right)<\hat{x}^{\mathcal{H}} C \hat{x},
$$

and we can choose a sufficiently small $\delta>0$, such that

$$
p_{*}+\delta\left(x_{*}^{\prime}\right)^{\mathcal{H}}\left(x_{*}^{\prime}\right)<\hat{x}^{\mathcal{H}} C \hat{x} \leq r_{*}^{(\delta)} .
$$

This follows from equations (23) and (24). For this $\delta,\left[\left(x_{*}^{\prime}\right)\left(x_{*}^{\prime}\right)^{\mathcal{H}}+\left(x_{*}^{(\delta)}\right)\left(x_{*}^{(\delta)}\right)^{\mathcal{H}}\right] / 2$ is a feasible point of $R P^{(\delta)}$ and satisfies

$$
\begin{aligned}
r_{*}^{(\delta)} & \leq \operatorname{tr}\left[(C+\delta \mathcal{I})\left(\frac{\left(x_{*}^{\prime}\right)\left(x_{*}^{\prime}\right)^{\mathcal{H}}+\left(x_{*}^{(\delta)}\right)\left(x_{*}^{(\delta)}\right)^{\mathcal{H}}}{2}\right)\right] \\
& =\frac{1}{2} r_{*}^{(\delta)}+\frac{1}{2}\left[p_{*}+\delta\left(x_{*}^{\prime}\right)^{\mathcal{H}}\left(x_{*}^{\prime}\right)\right] \\
& <r_{*}^{(\delta)} .
\end{aligned}
$$

This is a contradiction and hence $p_{*}=\hat{x}^{\mathcal{H}} C \hat{x}$.

Now, we show how to use this perturbation technique to solve $P$ in polynomial time. Solve $R P$ to get $p_{*}=r_{*}$. If the optimizer $W_{*}$ of $R P$ has rank at most 1 , compute $x_{*}$ from $W_{*}$ as in lemma 3 then we have solved $P$ in polynomial time. If it does not satisfy the rank condition, 
then choose an arbitrary $\delta_{0}>0$ and solve $R P^{\left(\delta_{0}\right)}$ in polynomial time to get the minimizer $W_{*}^{\left(\delta_{0}\right)}$ and the minimum $r_{*}^{\left(\delta_{0}\right)}=p_{*}^{\left(\delta_{0}\right)}$. For any $\delta$ in $\left(0, \delta_{0}\right)$,

$$
p_{*}=r_{*} \leq \operatorname{tr}\left(C W_{*}^{(\delta)}\right) \leq p_{*}^{(\delta)} .
$$

Also, $p_{*}^{(\delta)}$ is convex in $\delta[41]$ and hence

$$
p_{*}^{(\delta)} \leq p_{*}+\frac{\delta}{\delta_{0}}\left(p_{*}^{\left(\delta_{0}\right)}-p_{*}\right) .
$$

Given $\zeta>0$, choose $\delta$ sufficiently small so that $\frac{\delta}{\delta_{0}}\left(p_{*}^{\left(\delta_{0}\right)}-p_{*}\right) \leq \zeta$. For this $\delta$, solve $R P^{(\delta)}$ arbitrarily closely in polynomial time to get $W_{*}^{(\delta)}$ that has rank at most 1 and compute $x_{*}^{(\delta)}$. From equations (25) and (26), $x_{*}^{(\delta)}$ satisfies

$$
\left|\left(x_{*}^{(\delta)}\right)^{\mathcal{H}} C\left(x_{*}^{(\delta)}\right)-p_{*}\right| \leq \zeta .
$$

Thus $x_{*}^{(\delta)}$ is a feasible point of $P$ that has a value of the objective function within $\zeta$ of the theoretical optimum and we have shown a polynomial time algorithm to compute it.

This completes the proof of Theorem 1 .

\section{Matrices involved in OPF:}

Here we compute the $(i, j)$-th entries of $\left\{C_{k}, k \in \mathcal{K}\right\}$ for $O P F$. From (14), (15), (16), we have the following relations for $k \in[n]$, and $(p, q)$ and $(i, j)$ in $\mathcal{T}$ :

$$
\begin{aligned}
& {\left[\Phi_{k}\right]_{i j}= \begin{cases}\frac{1}{2} Y_{i j}=\frac{1}{2}\left(-g_{i j}+\mathbf{i} b_{i j}\right) & \text { if } k=i \\
\frac{1}{2} Y_{i j}^{\mathcal{H}}=\frac{1}{2}\left(-g_{i j}-\mathbf{i} b_{i j}\right) & \text { if } k=j \\
0 & \text { otherwise }\end{cases} } \\
& {\left[\Psi_{k}\right]_{i j}= \begin{cases}\frac{-1}{2 \mathbf{i}} Y_{i j}=\frac{1}{2}\left(-b_{i j}-\mathbf{i} g_{i j}\right) & \text { if } k=i \\
\frac{1}{2 \mathbf{i}} Y_{i j}^{\mathcal{H}}=\frac{1}{2}\left(-b_{i j}+\mathbf{i} g_{i j}\right) & \text { if } k=j \\
0 & \text { if } i=j=p\end{cases} } \\
& {\left[M^{p q}\right]_{i j}= \begin{cases}g_{p q} & \text { if }(i, j)=(p, q) \\
\frac{1}{2}\left(-g_{p q}+\mathbf{i} b_{p q}\right) & \text { if }(i, j)=(q, p) \\
\frac{1}{2}\left(-g_{p q}-\mathbf{i} b_{p q}\right) & \text { otherwise }, \\
0 & \end{cases} }
\end{aligned}
$$




$$
\left[T^{p q}\right]_{i j}= \begin{cases}g_{p q} & \text { if } i=j=p \text { or } i=j=q \\ -g_{p q} & \text { if }(i, j)=(p, q) \text { or }(i, j)=(q, p) \\ 0 & \text { otherwise. }\end{cases}
$$

\section{REFERENCES}

[1] F. Alizadeh, "Interior point methods in semidefinite programming with applications to combinatorial optimization," SIAM J. on Optimization, vol. 5, no. 1, pp. 13-51, 1995.

[2] Y. Nesterov and A. Nemirovskii, Interior-point polynomial algorithms in convex programming. Society for Industrial Mathematics, 1987, vol. 13.

[3] _- "Polynomial barrier methods in convex programming," Ekonom. i Mat. Metody, vol. 24, no. 6, pp. 1084-1091, 1988.

[4] Z. Luo, W. Ma, A. So, Y. Ye, and S. Zhang, "Semidefinite relaxation of quadratic optimization problems," Signal Processing Magazine, IEEE, vol. 27, no. 3, pp. 20 -34, May 2010.

[5] H. Wolkowicz, R. Saigal, and L. Vandenberghe, Handbook of Semidefinite Programming: Theory, Algorithms, and Applications. Springer Netherlands, 2000, vol. 27.

[6] A. Gershman, N. Sidiropoulos, S. Shahbazpanahi, M. Bengtsson, and B. Ottersten, "Convex optimization-based beamforming," Signal Processing Magazine, IEEE, vol. 27, no. 3, pp. 62-75, 2010.

[7] N. Sidiropoulos, T. Davidson, and Z. Luo, "Transmit beamforming for physical-layer multicasting," IEEE Trans.on Signal Processing, vol. 54, no. 6, pp. 2239-2251, 2006.

[8] A. So, Y. Ye, and J. Zhang, "A unified theorem on SDP rank reduction,” Mathematics of Operations Research, vol. 33, no. 4, pp. 910-920, 2008.

[9] Y. Huang and D. Palomar, "Rank-constrained separable semidefinite programming with applications to optimal beamforming," IEEE Trans. on Signal Processing, vol. 58, no. 2, pp. 664-678, 2010.

[10] P. Biswas, T. Lian, T. Wang, and Y. Ye, "Semidefinite programming based algorithms for sensor network localization," ACM Trans. on Sensor Networks (TOSN), vol. 2, no. 2, pp. 188-220, 2006.

[11] M. McCoy and J. Tropp, “Two proposals for robust PCA using semidefinite programming," Electronic J. of Statistics, vol. 5, pp. 1123-1160, 2011.

[12] A. Ljapunov and É. Davaux, Problème général de la stabilité du mouvement. Princeton Univ. Press, 1949.

[13] S. Boyd, L. El-Ghaoui, E. Feron, V. Balakrishnan, and E. Yaz, "Linear matrix inequalities in system and control theory," Proce. of the IEEE, vol. 85, no. 4, pp. 698-699, 1997.

[14] S. Boyd, L. Vandenberghe et al., "Semidefinite programming relaxations of non-convex problems in control and combinatorial optimization," Comm., Computation, Ctrl. and Signal Processing: A Tribute to Thomas Kailath, pp. 279-288, 1997.

[15] S. Kim and M. Kojima, "Exact solutions of some nonconvex quadratic optimization problems via SDP and SOCP relaxations," Computational Optimization and Applications, vol. 26, no. 2, pp. 143-154, 2003.

[16] S. Zhang, "Quadratic maximization and semidefinite relaxation," Mathematical Programming, vol. 87, no. 3, pp. 453-465, 2000.

[17] M. Goemans and D. Williamson, "Improved approximation algorithms for maximum cut and satisfiability problems using semidefinite programming," J. of the ACM (JACM), vol. 42, no. 6, pp. 1115-1145, 1995. 
[18] A. Frieze and M. Jerrum, "Improved approximation algorithms for max-cut and max bisection," Algorithmica, vol. 18, no. 1, pp. 67-81, 1997.

[19] D. Karger, R. Motwani, and M. Sudan, “Approximate graph coloring by semidefinite programming," J. of the ACM (JACM), vol. 45, no. 2, pp. 246-265, 1998.

[20] Y. Ye, "Approximating quadratic programming with bound and quadratic constraints," Mathematical Programming, vol. 84, no. 2, pp. 219-226, 1999.

[21] I. Nesterov, U. catholique de Louvain (1970-). Center for Operations Research, and Econometrics, Quality of semidefinite relaxation for nonconvex quadratic optimization. Center for Operations Research \& Econometrics. Université catholique de Louvain, 1997.

[22] Y. Nesterov, "Semidefinite relaxation and nonconvex quadratic optimization," Optimization methods and software, vol. 9, no. 1-3, pp. 141-160, 1998.

[23] J. Carpentier, "Contribution to the economic dispatch problem," Bulletin de la Societe Francoise des Electriciens, vol. 3, no. 8, pp. 431-447, 1962, in French.

[24] J. A. Momoh, Electric Power System Applications of Optimization, ser. Power Engineering, H. L. Willis, Ed. Markel Dekker Inc.: New York, USA, 2001.

[25] M. Huneault and F. D. Galiana, "A survey of the optimal power flow literature," IEEE Trans. on Power Systems, vol. 6, no. 2, pp. 762-770, 1991.

[26] J. A. Momoh, M. E. El-Hawary, and R. Adapa, "A review of selected optimal power flow literature to 1993. Part I: Nonlinear and quadratic programming approaches," IEEE Trans. on Power Systems, vol. 14, no. 1, pp. 96-104, 1999.

[27] — - "A review of selected optimal power flow literature to 1993. Part II: Newton, linear programming and interior point methods," IEEE Trans. on Power Systems, vol. 14, no. 1, pp. 105 - 111, 1999.

[28] K. S. Pandya and S. K. Joshi, “A survey of optimal power flow methods," J. of Theo. and Applied Info. Tech., vol. 4, no. 5 , pp. $450-458,2008$.

[29] X. Bai, H. Wei, K. Fujisawa, and Y. Wang, "Semidefinite programming for optimal power flow problems," Int'l J. of Electrical Power \& Energy Systems, vol. 30, no. 6-7, pp. 383-392, 2008.

[30] X. Bai and H. Wei, "Semi-definite programming-based method for security-constrained unit commitment with operational and optimal power flow constraints," IET Generation, Transmission \& Distribution, vol. 3, no. 2, pp. 182-197, 2009.

[31] J. Lavaei and S. Low, "Zero duality gap in optimal power flow problem," IEEE Trans. on Power Systems, To Appear, vol. 27, no. 1, pp. 92-107, 2012.

[32] S. Bose, D. F. Gayme, S. H. Low, and K. M. Chandy, "Optimal power flow over tree networks," in Proc. of the Allerton Conf. on Comm., Ctrl. and Computing, 2011.

[33] B. Zhang and D. Tse, "Geometry of feasible injection region of power networks," in Proc. of the Allerton Conf. on Comm., Ctrl. and Computing, 2011.

[34] S. Sojoudi and J. Lavaei, "Physics of power networks makes hard optimization problems easy to solve," in IEEE Power \& Energy Society (PES) General Meeting, July 2012.

[35] A. Ben-Tal and A. Nemirovski, "Lectures on modern convex optimization," Technion-Israel Institute of Technology, 2000.

[36] S. Boyd and L. Vandenberghe, Convex Optimization. Cambridge Univ. Press, 2004.

[37] Y. C. de Verdi 'ere, "Multiplicities of eigenvalues and tree-width graphs," J. of Combinatorial Theory, vol. 74, p. 121146, 1998, series B. 
[38] H. van der Holst, "Graphs whose positive semidefinite matrices have nullity at most two," Linear Algebra and its Applications, vol. 375, pp. 1-11, 2003.

[39] C. Johnson, A. Leal Duarte, C. Saiago, B. Sutton, and A. Witt, "On the relative position of multiple eigenvalues in the spectrum of an Hermitian matrix with a given graph," Linear Algebra and its Applications, vol. 363, pp. 147-159, 2003.

[40] J. Bonnans and A. Shapiro, "Optimization problems with perturbations: A guided tour," SIAM review, vol. 40, no. 2, pp. 228-264, 1998.

[41] E. Yıldırım and M. Todd, "Sensitivity analysis in linear programming and semidefinite programming using interior-point methods," Mathematical Programming, vol. 90, no. 2, pp. 229-261, 2001.

[42] R. Horn and C. Johnson, Matrix Analysis. Cambridge Univ. Press, 2005.

[43] G. Dantzig, A. Orden, and P. Wolfe, "The generalized simplex method for minimizing a linear form under linear inequality restraints," Pacific Journal of Mathematics, vol. 5, no. 2, pp. 183-195, 1955.

[44] G. Torres and V. Quintana, "An interior-point method for nonlinear optimal power flow using voltage rectangular coordinates," Power Systems, IEEE Transactions on, vol. 13, no. 4, pp. 1211-1218, 1998.

[45] I. Nejdawi, K. Clements, and P. Davis, "An efficient interior point method for sequential quadratic programming based optimal power flow," Power Systems, IEEE Transactions on, vol. 15, no. 4, pp. 1179-1183, 2000.

[46] R. Jabr, "A primal-dual interior-point method to solve the optimal power flow dispatching problem," Optimization and Engineering, vol. 4, no. 4, pp. 309-336, 2003.

[47] U. of Washington, "Power systems test case archive." [Online]. Available: http://www.ee.washington.edu/research/pstca/

[48] J. Lavaei, "Zero duality gap for classical opf problem convexifies fundamental nonlinear power problems," in Proc. of the American Ctrl. Conf., 2011.

[49] B. Lesieutre, D. Molzahn, A. Borden, and C. L. DeMarco, "Examining the limits of the application of semidefinite programming to power flow problems," in Proc. of the Allerton Conf. on Comm., Ctrl. and Computing, 2011.

[50] I. A. Hiskens and R. Davy, "Exploring the power flow solution space boundary," IEEE Trans. on Power Systems, vol. 16, no. 3, pp. 389-395, 2001.

[51] B. C. Lesieutre and I. A. Hiskens, "Convexity of the set of feasible injections and revenue adequacy in FTR markets," IEEE Trans. on Power Systems, vol. 20, no. 4, pp. 1790-1798, 2005.

[52] Y. V. Makarov, Z. Y. Dong, and D. J. Hill, "On convexity of power flow feasibility boundary," IEEE Trans. on Power Systems, vol. 23, no. 2, pp. 811-813, May 2008.

[53] M. E. Baran and F. F. Wu, "Optimal capacitor placement on radial distribution systems," IEEE Trans. on Power Delivery, vol. 4, no. 1, pp. 725-734, 1989.

[54] — - "Optimal sizing of capacitors placed on a radial distribution system," IEEE Trans. on Power Delivery, vol. 4, no. 1, pp. 735-743, 1989.

[55] J. A. Taylor, "Conic optimization of electric power systems," Ph.D. dissertation, MIT, June 2011.

[56] R. Jabr, "Radial distribution load flow using conic programming," IEEE Trans. on Power Systems, vol. 21, no. 3, pp. 1458-1459, Aug. 2006.

[57] M. Farivar, C. R. Clarke, S. H. Low, and K. M. Chandy, "Inverter VAR control for distribution systems with renewables," in Int'l Conf. on Smart Grid Comm. (SmartGridComm), 2011.

[58] L. Gan, N. Li, U. Topcu, and S. H. Low, "Branch flow model for radial networks: convex relaxation," in 51st IEEE Conference on Decision and Control, December 2012. 
[59] N. Li, L. Chen, and S. H. Low, "Exact convex relaxation of OPF for radial networks using branch flow model," in IEEE International Conference on Smart Grid Communications, November 2012.

[60] M. Farivar and S. H. Low, "Branch flow model: relaxations and convexification," in 51st IEEE Conference on Decision and Control, December 2012.

[61] S. Bose, S. H. Low, and K. M. Chandy, "Equivalence of branch flow and bus injection models," in Proc. of the Allerton Conf. on Comm., Ctrl. and Computing, October 2012.

[62] J. Lofberg, "YALMIP: A toolbox for modeling and optimization in MATLAB," in IEEE Int'l Symposium on Computer Aided Ctrl. Systems Design. IEEE, 2004, pp. 284-289.

[63] K. P. Schneider, Y. Chen, D. P. Chassin, R. Pratt, D. Engel, and S. Thompson, "Modern grid initiative: Distribution taxonomy final report," U.S. Department of Energy, Tech. Rep., 2008.

[64] K. Turitsyn, P. Sŭlc, S. Backhaus, and M. Chertkov, "Options for control of reactive power by distributed photovoltaic generators," Proc. of the IEEE, vol. 99, no. 6, pp. 1063 -1073, June 2011.

[65] D. F. Yeh, H.-G.and Gayme and S. H. Low, "Adaptive VAR control for distribution circuits with photovoltaic generator," IEEE Trans. on Power Systems, to appear, 2012. 